\title{
Prevalence and time trends of refractive error in Chinese children: A systematic review and meta-analysis
}

Yi Tang ${ }^{1,2 *}$, Aiming Chen ${ }^{3 *}$, Minjie Zou $^{1 *}$, Zhenzhen Liu' ${ }^{1}$, Charlotte Aimee Young ${ }^{4}$, Danying Zheng ${ }^{1}$, Guangming $\operatorname{Jin}^{1}$

${ }^{1}$ State Key Laboratory of Ophthalmology, Zhongshan Ophthalmic Center, Sun Yat-Sen University, Guangzhou, China

${ }^{2}$ Zhongshan School of Medicine, Sun Yat-sen University, Guangzhou, China

${ }^{3}$ Department of Pharmacy, The Fifth

Affiliated Hospital of Sun Yat-Sen University, Zhuhai, China

${ }^{4}$ Nanchang Eye Hospital, Third Affiliated Hospital of Nanchang University, Nanchang, China

*Joint first authors.
Background To investigate the prevalence and time trends of refractive error (RE) among Chinese children under 18 years old.

Methods PubMed, Embase, Web of Science were searched for articles that estimated prevalence of RE in Chinese children. Data of identified eligible studies was extracted by two investigators independently. Pooled prevalence of RE and its $95 \%$ confidence interval (95\% CI) and the time trends of RE were investigated using Meta-analysis methods.

Results Of the 41 studies covering 1051784 subjects, the pooled prevalence of myopia, high myopia, hyperopia and astigmatism in Chinese children was $38.0 \%$ (95\% confidence interval $(\mathrm{CI})=35.1 \%-41.1 \%), 2.8 \%(95 \% \mathrm{CI}=2.3 \%$ $3.4 \%$ ), $5.2 \%$ (95\% CI=3.1\%-8.6\%) and $16.5 \%$ (95\% CI=12.3\%-21.8\%), respectively. Subgroup analysis show that children living in urban were at higher risk of RE. Prevalence of myopia and hyperopia were higher in Northern China compared with Southern China and high myopia and astigmatism were higher in Hong Kong, Macau and Taiwan than in mainland China. Regression analysis showed an upward trend in myopia and hyperopia and a downward trend in high myopia and astigmatism with years.

Conclusions The prevalence of RE is higher in urban areas than in rural for Chinese children. The much higher prevalence of myopia and astigmatism in China compared with foreign countries indicates the important role played by environment and genetic factors. Considering the large magnitude of refractive errors, much more attention should still be paid to RE prevention and treatment strategy development in China.

Refractive error (RE) has been one of the most common eye disorders among children and adolescents and one of the major public health concerns in the world. It has been reported that $42 \%$ of visual impairments are caused by RE globally [1]. $\mathrm{RE}$ have profound effects on children, for not only will it increase the possibility of pathologic ocular changes such as myopic macular degeneration and retinal detachment, which could lead to irreversible blindness, but it also has a great impact on psychosocial well-being for children, which can limit their educational outcomes and educational opportunities [2-4].

In East Asia, the high prevalence of RE has been a major public health concern. For urban areas of these countries, about $80 \%$ of the adolescents in high school are myopic, while $10 \%-20 \%$ of them suffer from high myopia [5]. Also, it is reported that the prevalence of hyperopia and astigmatism in Asian children was $4.6 \%$ and 
$14.9 \%$ respectively [6]. Moreover, striking evidence suggests a growing trend of RE prevalence especially among young East Asians [7,8]. It is expected that by 2050, 4758 million people will be myopic and 938 million people will suffer from high myopia globally [9]. China, the most populous country in the world that accounting for one fifth of world population, has had a high prevalence of RE during the past decades and possessing a large number of RE patients $[6-8,10]$.

Considering the impact of RE and its high prevalence, it is undeniable that there is great value in further understanding the epidemiology of RE for the purposes of policy making. Particularly, policy of myopia prevention and control has been a hotspot in the field of public health since the rapid rise of prevalence of myopia in China. Although numerous population-based or school-based studies and meta-analysis have been performed to evaluate the prevalence of RE in China, most of them focus on myopia and high myopia [10-13], which does not shed light on the magnitude and time trend of total RE, especially hyperopia and astigmatism, among the young Chinese population and there is a lack of study reporting the epidemiologic characteristics of RE as a whole.

Considering an overall estimate of the magnitude and its time trends of RE is important for RE prevent and control, we performed this meta-analysis and comprehensive systematic review to evaluate the prevalence of refractive errors, time trends, and its sub-classifications among children in China, which might provide useful information for appropriate preventive strategies to reduce the disease burden caused by SE in China and beyond.

\section{METHODS}

\section{Search strategy}

The protocol of the meta-analysis was registered in PROSPERO website (University of York, United Kingdom) with a registration number of CRD42020197708. In order to extract articles providing prevalence data of refractive errors in Chinese children, bibliographic databases including PubMed, EMBASE, and Web of Science were searched with different combinations of words including

1. Population: "China", "Chinese", "Taiwan", "Macau", "Macao", "Hong Kong”

2. Outcome: "refractive errors", "myopia", "astigmatism", "hyperopia"

3. Study design: "Prevalence", "Epidemiology", "epidemiology", "prevalence", "incidence"

The search was conducted by two investigators (TY, ZMJ) independently with the final search date of July 28 , 2019 .

\section{Study selection}

After the search, 4240 articles were identified. 1641 duplicate articles were removed. The selection was conducted by two investigators independently with the following criteria:

The inclusion criteria were as follows: 1) school-based studies or population-based studies with clearly defined sampling strategies; 2) studies reporting the prevalence of refractive errors in Chinese children younger than 18 years old; 3) studies with a clear definition of refractive errors; 4) sample size of at least 1000 subjects. Studies with sample size less than 1000 were excluded because it's age-defined subgroups would be too small for a reliable assessment of the prevalence of refractive errors.

The exclusion criteria were as follows: 1) hospital-based or clinical-based surveys; 2) conducted only in a single school; 3) using visual acuity as the measurement for refractive errors; 4) missing or incomplete data; 5) obvious limitations in their statistical analysis or design; 6) different studies based on the same population without providing additional information.

\section{Data extraction}

The searches were limited to English language literature only. After the selection procedure, 41 articles that met the inclusion criteria were carefully reviewed by two investigators (TY, ZMJ). The extracted data of these articles are listed as follows:

1) Characteristics of the study: author, study year, design of study, refraction with or without mydriatics.

2) Characteristics of the studied population: sample size, age range, district and region (urban or rural) of the sample, percentage of female subjects.

3) Prevalence data: definition and prevalence of refractive errors. 


\section{Statistical analysis}

Pooled prevalence of refractive error and its 95\% confidence interval (95\% CI) was estimated. Subgroup analyses were conducted for potential difference in region and study year. The I-square test was performed to estimate the heterogeneity of the included studies ( $<50 \%$ indicates low heterogeneity, and $>50 \%$ presents high degree of heterogeneity). When the I-square test suggested a high degree of heterogeneity, a random effect model was used, otherwise a fixed effect model was used.[10] To access the publication bias of these studies, Egger's tests and Begg's tests were performed and the significance level was set at $P<0.05$ (2-tailed). Funnel plots were also performed for publication bias when more than 10 studies were involved in the meta-analysis. Sensitivity analysis of studies included in myopia, high myopia, hyperopia, astigmatism were conducted. Time trends of refractive errors were also investigated by meta regression. This meta-analysis was performed with the Comprehensive Meta-Analysis Software V.2 (Biostat, Englewood, New Jersey, USA).

\section{RESULTS}

Figure 1 shows the process of literature selection. 4240 records were identified by literature research. After the selection, 41 studies including 1051784 subjects were included for qualitative synthesis [11-51]. Among these studies, 15 studies were conducted in Northern China including 196547 subjects (18.7\%) [11,13,17,2 $2,23,25,28,33,34,37,38,40,41,48,49] ; 19$ studies with 98885 participants (9.4\%) were conducted in Southern China [14-16,18,19,21,24,29-32,35,36,39,42,43,45-47]; 6 studies including 89213 subjects (8.5\%) were launched in Hong Kong, Macao and Taiwan (HMT), [12,20,26,27,44,50], and 1 multicenter study included 667139 individuals (63.4\%) [51]. The basic characteristics of these studies are shown in Table 1 and the prevalence and definitions of RE are given in Table 2.

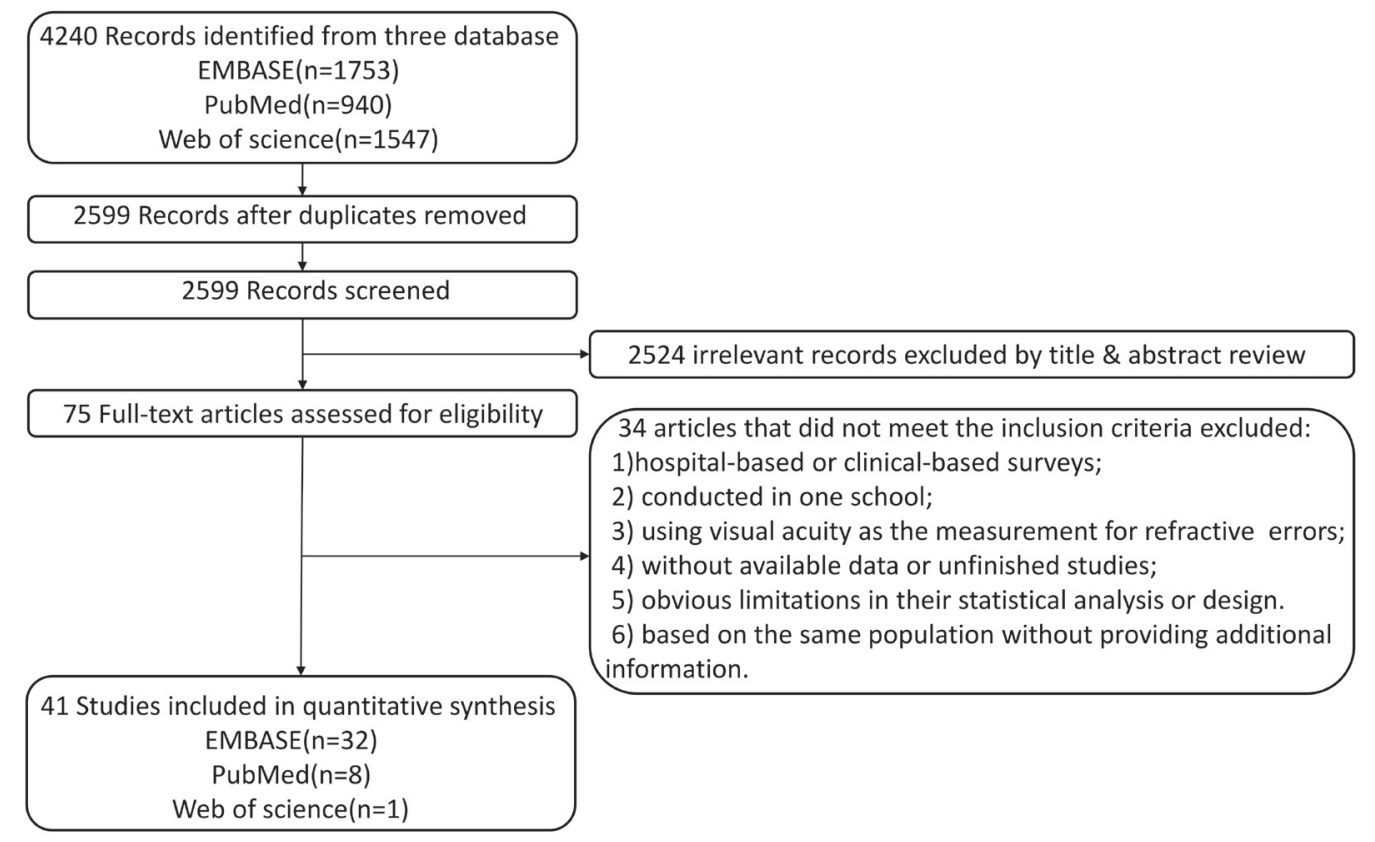

Figure 1. Flowchart of the study selection process.

\section{Prevalence of myopia and high myopia}

As is shown in Table 3 and Figure 2, Panel A, the pooled prevalence of myopia was 38.0\% (95\% CI=35.1\%41.1\%). Urban children had a significantly higher prevalence of myopia than rural children: $51.2 \%$ (95\% $\mathrm{CI}=50.8 \%-51.5 \%)$ vs $27.1 \%(95 \% \mathrm{CI}=26.7 \%-27.5 \%) ; \mathrm{P}<0.001$. Additionally, pooled prevalence in HMT and Northern China were similar: $53.0 \%$ (95\% CI=52.6-53.4\%) vs 55.1\% (95\% CI=54.9\%-55.3\%), while Southern China hadhas the lowest prevalence: $31.4 \%$ (95\% CI=31.0\%-31.7\%), $P<0.001$. The subgroup analysis of study year shows that there was a higher prevalence before the year 2000: 49.3\% (95\% CI=37.9\%60.7\%). However, as the regression analysis shows (Figure S1 in the Online Supplementary Document), there WAS a slight increasing trend of the prevalence of myopia (equation of the regression line: myopia prevalence $(\%)=0.00824 \times$ midpoint of the study year group $-16.47958 ; P<0.01)$. 
Table 1. The basic characteristics of included studies

\begin{tabular}{|c|c|c|c|c|c|c|c|c|c|}
\hline AUTHOR & STUDY YEAR & DISTRICT & REGION & DESIGN OF STUDY & SAMPLE SIZE & AGE(MEAN OR RANGE) & GIRLS & REFRACTION & CYCLOPIEGIA \\
\hline Guo et al. [14] & NA & SC & $\mathrm{U}$ and $\mathrm{R}$ & SB & 5182 & $3-6$ & NA & $\mathrm{AU}$ & yes \\
\hline Guo et al. [15] & 2014 & SC & $\mathrm{U}$ & SB & 3055 & $7-15$ & 48.3 & $\mathrm{AU}$ & yes \\
\hline Han et al. [16] & 2015 & SC & $\mathrm{U}$ & SB & 8662 & $5-16$ & 45.17 & $\mathrm{AU}$ & yes \\
\hline Li et al. [22] & 2013 & $\mathrm{NC}$ & $\mathrm{U}$ & SB & 1839 & $12.9-17.6$ & 51.6 & $\mathrm{AU}$ & yes \\
\hline Li et al. [24] & 2013 & SC & $\mathrm{U}$ & SB & 7166 & $4-6$ & 46.8 & $\mathrm{AU}$ & no \\
\hline \multirow[t]{4}{*}{ Lin et al. [26] } & 1983 & HMT & $\mathrm{U}$ and $\mathrm{R}$ & SB & 4125 & $7-18$ & NA & $\mathrm{AU}$ & yes \\
\hline & 1986 & HMT & $\mathrm{U}$ and $\mathrm{R}$ & SB & 10500 & $7-18$ & $\mathrm{NA}$ & $\mathrm{AU}$ & yes \\
\hline & 1990 & HMT & $\mathrm{U}$ and $\mathrm{R}$ & SB & 8667 & $7-18$ & $\mathrm{NA}$ & $\mathrm{AU}$ & yes \\
\hline & 1995 & HMT & $\mathrm{U}$ and $\mathrm{R}$ & SB & 11178 & $7-18$ & 49.2 & $\mathrm{AU}$ & $\mathrm{NA}$ \\
\hline Congdon et al. [43] & 2007 & $\mathrm{SC}$ & $\mathrm{R}$ & SB & 1892 & $11.4-17.1$ & 51.2 & $\mathrm{AU}$ & yes \\
\hline Lin et al. [27] & 2000 & HMT & $\mathrm{U}$ and $\mathrm{R}$ & SB & 10889 & $7-18$ & 48 & $\mathrm{AU}$ & yes \\
\hline Lyu et al. [28] & 2011 & $\mathrm{NC}$ & $\mathrm{U}$ & SB & 4249 & $5-14$ & 48.2 & $\mathrm{AU}$ & yes \\
\hline Ma et al. [29] & 2013 & SC & $\mathrm{R}$ & SB & 5532 & $3-10$ & 45.3 & $\mathrm{AU}$ & yes \\
\hline Qian et al. [33] & 2017 & $\mathrm{NC}$ & $\mathrm{U}$ & SB & 8683 & $6-18$ & 45.7 & $\mathrm{AU}$ & no \\
\hline Guo et al. [11] & 2016 & NC & $\mathrm{U}$ and $\mathrm{R}$ & SB & 35745 & $6-18$ & 48.9 & $\mathrm{AU}$ & no \\
\hline Han et al. [17] & 2013 & NC & $\mathrm{R}$ & SB & 2147 & 11-15 & 48.1 & $\mathrm{AU}$ & NA \\
\hline Li et al. [25] & $2008-2009$ & $\mathrm{NC}$ & $\mathrm{R}$ & $\mathrm{PB}$ & 1675 & $5-18$ & 46.1 & $\mathrm{AU}$ & yes \\
\hline \multirow[t]{2}{*}{ Pan et al. [30] } & 2016 & SC & $\mathrm{R}$ & $\mathrm{SB}$ & 2432 & mean 7.7 & 44.8 & $\mathrm{AU}$ & yes \\
\hline & 2016 & SC & $\mathrm{R}$ & SB & 2346 & mean 13.8 & 48.3 & $\mathrm{AU}$ & yes \\
\hline Pi et al. [31] & $2006-2007$ & SC & $\mathrm{R}$ & $\mathrm{PB}$ & 3070 & $6-15$ & 47.5 & $\mathrm{RE}$ & yes \\
\hline Pi et al. [32] & 2006 & SC & $\mathrm{R}$ & $\mathrm{PB}$ & 3079 & $6-15$ & 47.5 & $\mathrm{RE}$ & $\mathrm{NA}$ \\
\hline Shi et al. [34] & NA & $\mathrm{NC}$ & $\mathrm{U}$ & $\mathrm{SB}$ & 2046 & 7 to 12 & 46.1 & $\mathrm{AU}$ & no \\
\hline \multirow[t]{2}{*}{ Wang et al. [35] } & 2009 & SC & $\mathrm{U}$ and $\mathrm{R}$ & SB & 1235 & $13-15$ & 51.2 & $\mathrm{AU}$ & $\mathrm{NA}$ \\
\hline & 2009 & SC & $\mathrm{U}$ and $\mathrm{R}$ & SB & 1183 & $16-18$ & 42.8 & $\mathrm{AU}$ & $\mathrm{NA}$ \\
\hline Wang et al. [36] & 2011 & SC & $\mathrm{U}$ & SB & 2255 & 2-6.7 (24-80 months) & 44.7 & $\mathrm{RE}$ & yes \\
\hline Wu et al. [37] & NA & $\mathrm{NC}$ & $\mathrm{R}$ & SB & 6026 & $4-18$ & 47.1 & $\mathrm{AU}$ & yes \\
\hline Wu et al. [38] & $\mathrm{NA}$ & $\mathrm{NC}$ & $\mathrm{U}$ and $\mathrm{R}$ & SB & 4677 & $15-18$ & 53.7 & $\mathrm{AU}$ & no \\
\hline Hsu et al. [20] & 2013 & HMT & $\mathrm{U}$ and $\mathrm{R}$ & $\mathrm{PB}$ & 11590 & 8 (grade 2) & 47.1 & $\mathrm{AU}$ & yes \\
\hline Hu et al. [21] & 2014 & SC & $\mathrm{R}$ & SB & 10037 & $9-12$ & 47.7 & $\mathrm{AU}$ & no \\
\hline Lam et al. [12] & $2005-2010$ & HMT & $\mathrm{U}$ & SB & 2651 & $5-15$ & 46.8 & $\mathrm{AU}$ & no \\
\hline \multirow[t]{2}{*}{ Li et al. [23] } & 2011-2012 & $\mathrm{NC}$ & $\mathrm{U}$ & SB & 2893 & $5.7-9.3$ & 42.2 & $\mathrm{AU}$ & yes \\
\hline & 2011-2012 & $\mathrm{NC}$ & $\mathrm{U}$ & SB & 2267 & $10.0-15.9$ & 50 & $\mathrm{AU}$ & yes \\
\hline Xia et al. [39] & 2009 & SC & $\mathrm{R}$ & SB & 3517 & 7-11 & 44.2 & $\mathrm{AU}$ & yes \\
\hline Yang et al. [40] & 2015 & $\mathrm{NC}$ & $\mathrm{U}$ and $\mathrm{R}$ & SB & 61036 & $7-18$ & 48.7 & $\mathrm{AU}$ & no \\
\hline Zeng et al. [42] & 2017 & SC & $\mathrm{U}$ and $\mathrm{R}$ & $\mathrm{SB}$ & 16955 & $6-10$ & 44.2 & $\mathrm{RE}$ & yes \\
\hline Zhao et al. [49] & 1998 & $\mathrm{NC}$ & $\mathrm{R}$ & $\mathrm{PB}$ & 5884 & $5-15$ & 48.9 & $\mathrm{AU}$ & yes \\
\hline Qian et al. [47] & 2014 & SC & $\mathrm{R}$ & SB & 7681 & $5-16$ & 49.3 & $\mathrm{AU}$ & yes \\
\hline \multirow[t]{3}{*}{ Dong et al. [51] } & 2005 & $\mathrm{M}$ & $\mathrm{U}$ and $\mathrm{R}$ & $\mathrm{PB}$ & 235505 & $7-18$ & 49.8 & $\mathrm{NA}$ & $\mathrm{NA}$ \\
\hline & 2010 & $\mathrm{M}$ & $\mathrm{U}$ and $\mathrm{R}$ & $\mathrm{PB}$ & 216474 & $7-18$ & 50 & $\mathrm{NA}$ & $\mathrm{NA}$ \\
\hline & 2014 & $\mathrm{M}$ & $\mathrm{U}$ and $\mathrm{R}$ & $\mathrm{PB}$ & 215160 & $7-18$ & 50 & $\mathrm{NA}$ & $\mathrm{NA}$ \\
\hline Fan et al. [44] & $1998-2000$ & HMT & $\mathrm{U}$ & SB & 7560 & $6-15$ & 49.5 & $\mathrm{AU}$ & yes \\
\hline He et al. [19] & 2002-2003 & $\mathrm{SC}$ & $\mathrm{U}$ & $\mathrm{PB}$ & 4364 & $5-15$ & 48.4 & $\mathrm{AU}$ and $\mathrm{RE}$ & yes \\
\hline Lan et al. [46] & 2009 & SC & $\mathrm{U}$ and $\mathrm{R}$ & SB & 2478 & $3-6$ & 47.2 & $\mathrm{AU}$ and $\mathrm{RE}$ & yes \\
\hline Sun et al. [48] & $2015-2016$ & $\mathrm{NC}$ & $\mathrm{U}$ & SB & 4890 & $10-15$ & 48.3 & $\mathrm{AU}$ & yes \\
\hline \multirow[t]{2}{*}{ Shih et al [50] } & 1995 & HMT & $\mathrm{U}$ and $\mathrm{R}$ & SB & 11175 & $7-18$ & $\mathrm{NA}$ & $\mathrm{AU}$ & yes \\
\hline & 2000 & HMT & $\mathrm{U}$ and $\mathrm{R}$ & $\mathrm{SB}$ & 10878 & $7-18$ & NA & $\mathrm{AU}$ & yes \\
\hline \multirow[t]{10}{*}{ Li et al. [13] } & 2006 & $\mathrm{NC}$ & $\mathrm{U}$ & SB & 3657 & $14-16$ & 52.37 & $\mathrm{AU}$ & yes \\
\hline & 2007 & $\mathrm{NC}$ & $\mathrm{U}$ & SB & 3615 & $14-16$ & 52.42 & $\mathrm{AU}$ & yes \\
\hline & 2008 & $\mathrm{NC}$ & $\mathrm{U}$ & SB & 3662 & $14-16$ & 52.81 & $\mathrm{AU}$ & yes \\
\hline & 2009 & $\mathrm{NC}$ & $\mathrm{U}$ & $\mathrm{SB}$ & 3697 & $14-16$ & 50.42 & $\mathrm{AU}$ & yes \\
\hline & 2010 & $\mathrm{NC}$ & $\mathrm{U}$ & SB & 3897 & $14-16$ & 52.45 & $\mathrm{AU}$ & yes \\
\hline & 2011 & $\mathrm{NC}$ & $\mathrm{U}$ & SB & 3784 & $14-16$ & 49.1 & $\mathrm{AU}$ & yes \\
\hline & 2012 & $\mathrm{NC}$ & $\mathrm{U}$ & SB & 3816 & $14-16$ & 54.09 & $\mathrm{AU}$ & yes \\
\hline & 2013 & $\mathrm{NC}$ & $\mathrm{U}$ & SB & 3787 & $14-16$ & 52.05 & $\mathrm{AU}$ & yes \\
\hline & 2014 & $\mathrm{NC}$ & $\mathrm{U}$ & SB & 3833 & $14-16$ & 53.48 & $\mathrm{AU}$ & yes \\
\hline & 2015 & $\mathrm{NC}$ & $\mathrm{U}$ & SB & 3676 & $14-16$ & 51.8 & $\mathrm{AU}$ & yes \\
\hline He et al. [45] & $2002-2003$ & SC & $\mathrm{U}$ & $\mathrm{PB}$ & 4364 & $5-15$ & 48.5 & $\mathrm{RE}$ & yes \\
\hline You et al. [41] & 2012 & $\mathrm{NC}$ & $\mathrm{U}$ and $\mathrm{R}$ & $\mathrm{PB}$ & 15066 & $7-18$ & 51.6 & $\mathrm{AU}$ & no \\
\hline He et al. [18] & 2005 & SC & $\mathrm{R}$ & SB & 2400 & $13-17$ & 49.1 & $\mathrm{AU}$ & yes \\
\hline
\end{tabular}

SC - Southern China, NC - Northern China, HMT - Hong Kong, Macao and Taiwan, M - multiple center, U - urban, R - rural, SB - school-based, PB - population-based, AU - auto refraction, RE - retinoscopy 
Table 2. Prevalence of refractive errors in the included studies

\begin{tabular}{|c|c|c|c|c|c|c|c|c|}
\hline \multirow{2}{*}{ AUTHOR } & \multicolumn{2}{|c|}{ MYOPIA } & \multicolumn{2}{|c|}{ HIGH MYOPIA } & \multicolumn{2}{|c|}{ HYPEROPIA } & \multicolumn{2}{|c|}{ AstigmATISM } \\
\hline & Definition & Prevalence & Definition & Prevalence & Definition & Prevalence & Definition & Prevalence \\
\hline Guo et al. (NA)* [14] & $S E \leq-0.5 \mathrm{D}$ & 1.95 & NA & NA & NA & NA & NA & NA \\
\hline Guo et al. (2014) [15] & $\mathrm{SE} \leq-0.5 \mathrm{D}$ & 47.3 & $\mathrm{SE} \leq-6.0 \mathrm{D}$ & 1.8 & NA & NA & NA & NA \\
\hline Guo et al. (2016) [11] & $\mathrm{SE} \leq-0.5 \mathrm{D}$ & 70.9 & $\mathrm{SE} \leq-6.0 \mathrm{D}$ & 8.6 & NA & NA & NA & NA \\
\hline Han et al. (2015) [16] & $\mathrm{SE} \leq-0.25 \mathrm{D}$ & 62.6 & $\mathrm{SE} \leq-6.0 \mathrm{D}$ & 1.3 & $\mathrm{SE} \geq 0.5 \mathrm{D}$ & 11.3 & $\mathrm{SE} \geq 0.5 \mathrm{D}$ & 11 \\
\hline Han et al. (2013) [17] & $\mathrm{SE} \leq-0.75 \mathrm{D}$ & 48.02 & $\mathrm{SE}<-6.0 \mathrm{D}$ & 11.5 & NA & $\mathrm{NA}$ & NA & NA \\
\hline Li et al. (2013) [22] & $\mathrm{SE} \leq-0.5 \mathrm{D}$ & 82.7 & NA & 7.1 & $\mathrm{SE} \geq 0.5 \mathrm{D}$ & 7.5 & NA & NA \\
\hline Li et al. (2013) [24] & $\mathrm{SE} \leq-1.0 \mathrm{D}$ & 5.9 & $\mathrm{SE} \leq-6.0 \mathrm{D}$ & 0.1 & $\mathrm{SE} \geq 2.0 \mathrm{D}$ & 1 & $\mathrm{SE} \geq 1.0 \mathrm{D}$ & 12.7 \\
\hline \multirow[t]{2}{*}{ Li et al. $(2011,2012)[23]$} & $\mathrm{SE} \leq-0.5 \mathrm{D}$ & 3.9 & $\mathrm{SE} \leq-6.0 \mathrm{D}$ & 0.1 & $\mathrm{SE} \geq 2.0 \mathrm{D}$ & 23.3 & $\mathrm{SE} \geq 0.75 \mathrm{D}$ & 25.6 \\
\hline & $\mathrm{SE} \leq-0.5 \mathrm{D}$ & 67.3 & $\mathrm{SE} \leq-6.0 \mathrm{D}$ & 2.7 & $\mathrm{SE} \geq 2.0 \mathrm{D}$ & 1.2 & $\mathrm{SE} \geq 0.75 \mathrm{D}$ & 28.3 \\
\hline Li et al. (2008) [25] & $\mathrm{SE} \leq-0.5 \mathrm{D}$ & 5 & NA & NA & $\mathrm{SE} \geq 0.5 \mathrm{D}$ & 1.6 & $S E \geq 0.75 D$ & 2 \\
\hline \multirow{4}{*}{$\begin{array}{l}\text { Lin et al. }(1983,1986 \text {, } \\
1990,1995)[26]\end{array}$} & $\mathrm{SE}<-0.25 \mathrm{D}$ & 62.1 & $\mathrm{SE}<-6.0 \mathrm{D}$ & 8.9 & NA & NA & NA & NA \\
\hline & $\mathrm{SE}<-0.25 \mathrm{D}$ & 57.5 & $\mathrm{SE}<-6.0 \mathrm{D}$ & 7 & NA & NA & NA & NA \\
\hline & $\mathrm{SE}<-0.25 \mathrm{D}$ & 56.8 & $\mathrm{SE}<-6.0 \mathrm{D}$ & 5.5 & NA & NA & NA & NA \\
\hline & $\mathrm{SE}<-0.25 \mathrm{D}$ & 63.2 & $\mathrm{SE}<-6.0 \mathrm{D}$ & 10.6 & NA & NA & NA & NA \\
\hline Lin et al. (2000) [27] & $\mathrm{SE}<-0.25 \mathrm{D}$ & 61.4 & $\mathrm{SE}<-6.0 \mathrm{D}$ & 7.1 & NA & NA & NA & $\mathrm{NA}$ \\
\hline Lyu et al. [28] & $\mathrm{SE} \leq-0.5 \mathrm{D}$ & 36.7 & NA & NA & $\mathrm{SE} \geq 2.0 \mathrm{D}$ & 2.4 & $\mathrm{SE} \geq 1.0 \mathrm{D}$ & 28.1 \\
\hline Qian et al. (2017) [33] & $\mathrm{SE} \leq-0.75 \mathrm{D}$ & 42.8 & NA & NA & NA & NA & NA & NA \\
\hline Qian et al. (2014) [47] & $\mathrm{SE}<-0.5 \mathrm{D}$ & 39.1 & $\mathrm{SE}<-6.0 \mathrm{D}$ & 0.6 & NA & NA & NA & NA \\
\hline Hsu et al. [20] & $\mathrm{SE} \leq-0.5 \mathrm{D}$ & 36.4 & NA & NA & NA & NA & NA & NA \\
\hline Hu et al.[21] & $\mathrm{SE} \leq-0.5 \mathrm{D}$ & 8 & NA & NA & $S E \geq 2.0 \mathrm{D}$ & NA & $\mathrm{SE} \geq 0.75 \mathrm{D}$ & NA \\
\hline Lam et al.[12] & $\mathrm{SE}<-0.5 \mathrm{D}$ & 47.5 & $\mathrm{SE}<-6.0 \mathrm{D}$ & 1.8 & $\mathrm{SE}>0.5 \mathrm{D}$ & 8.1 & NA & NA \\
\hline \multirow[t]{2}{*}{ Pan et al.[30] } & $\mathrm{SE}<-0.5 \mathrm{D}$ & 2.4 & $\mathrm{SE}<-6.0 \mathrm{D}$ & 0.1 & NA & NA & NA & NA \\
\hline & $\mathrm{SE}<-0.5 \mathrm{D}$ & 29.4 & $\mathrm{SE}<-6.0 \mathrm{D}$ & 0.4 & NA & NA & NA & NA \\
\hline Pi et al.(2006-2007)[31] & $\mathrm{SE} \leq-0.5 \mathrm{D}$ & 13.75 & NA & NA & $\mathrm{SE} \geq 1.5 \mathrm{D}$ & 12.51 & $\mathrm{SE} \geq 0.5 \mathrm{D}$ & 11.17 \\
\hline Pi et al.(2006)[32] & $\mathrm{SE} \leq-0.5 \mathrm{D}$ & 13.7 & NA & NA & $\mathrm{SE} \geq 2.0 \mathrm{D}$ & 3.3 & $\mathrm{SE} \geq 1.0 \mathrm{D}$ & 3.7 \\
\hline Shi et al.[34] & $\mathrm{SE} \leq-0.5 \mathrm{D}$ & 63.8 & $\mathrm{SE}<-6.0 \mathrm{D}$ & 1.1 & $\mathrm{SE}>0.5 \mathrm{D}$ & 4.2 & NA & NA \\
\hline Ma et al.[29] & $\mathrm{SE} \leq-0.5 \mathrm{D}$ & 20.1 & $\mathrm{SE} \leq-6.0 \mathrm{D}$ & 0.3 & $\mathrm{SE} \geq 2.0 \mathrm{D}$ & 11.4 & $\mathrm{SE} \leq-1.0 \mathrm{D}$ & 23 \\
\hline Congdon et al.[43] & $\mathrm{SE}<-0.5 \mathrm{D}$ & 62.3 & $\mathrm{SE}<-6.0 \mathrm{D}$ & 1.9 & $\mathrm{SE} \geq 2.0 \mathrm{D}$ & 0.2 & $\mathrm{SE}>0.75 \mathrm{D}$ & 1.7 \\
\hline Wu et al.(NA) [37] & $\mathrm{SE} \leq-0.5 \mathrm{D}$ & 36.9 & $\mathrm{SE} \leq-6.0 \mathrm{D}$ & 2 & $\mathrm{SE}>0.5 \mathrm{D}$ & 48.6 & $\mathrm{SE} \geq 0.75 \mathrm{D}$ & 36.3 \\
\hline Wu et al.(NA) [38] & $\mathrm{SE} \leq-1.0 \mathrm{D}$ & 80.7 & $\mathrm{SE} \leq-6.0 \mathrm{D}$ & 9.9 & NA & NA & NA & NA \\
\hline \multirow{2}{*}{ Wang et al.(2009)[35] } & $\mathrm{SE} \leq-0.75 \mathrm{D}$ & 48.07 & NA & NA & NA & NA & NA & NA \\
\hline & $\mathrm{SE} \leq-0.75 \mathrm{D}$ & 68.28 & NA & NA & NA & NA & NA & NA \\
\hline Wang et al.(2011)[36] & $\mathrm{SE} \leq-1.0 \mathrm{D}$ & 0.9 & NA & NA & $\mathrm{SE} \geq 2.0 \mathrm{D}$ & 14.3 & $\mathrm{SE} \geq 1.0 \mathrm{D}$ & 8.8 \\
\hline Xia et al.[39] & $S E<-0.5 \mathrm{D}$ & 14.5 & NA & NA & NA & NA & NA & NA \\
\hline Yang et al.[40] & $\mathrm{SE} \leq-0.5 \mathrm{D}$ & 49.8 & $\mathrm{SE} \leq-6.0 \mathrm{D}$ & 3 & NA & NA & NA & NA \\
\hline You et al.[41] & $\mathrm{SE} \leq-1.0 \mathrm{D}$ & 53 & $\mathrm{SE} \leq-6.0 \mathrm{D}$ & 4.3 & NA & NA & NA & NA \\
\hline Zeng et al.[42] & $\mathrm{SE} \leq-0.5 \mathrm{D}$ & 24.15 & $\mathrm{SE}<-6.0 \mathrm{D}$ & 0.64 & NA & NA & NA & NA \\
\hline \multirow[t]{3}{*}{ Dong et al.[51] } & $\mathrm{SE} \leq-0.5 \mathrm{D}$ & 47.4 & NA & NA & NA & NA & NA & NA \\
\hline & $\mathrm{SE} \leq-0.5 \mathrm{D}$ & 55.6 & NA & NA & NA & NA & NA & NA \\
\hline & $\mathrm{SE} \leq-0.5 \mathrm{D}$ & 57.2 & NA & NA & NA & NA & NA & NA \\
\hline Fan et al.[44] & $\mathrm{SE} \leq-0.5 \mathrm{D}$ & 36.71 & $\mathrm{SE} \leq-6.0 \mathrm{D}$ & 1.19 & $\mathrm{SE} \geq 2.0 \mathrm{D}$ & 4 & $\mathrm{SE} \geq 1.0 \mathrm{D}$ & 18.1 \\
\hline He et al.(2002) [45] & $\mathrm{SE} \leq-0.5 \mathrm{D}$ & 35.1 & NA & NA & $\mathrm{SE} \geq 2.0 \mathrm{D}$ & 5.8 & $\mathrm{SE} \geq 0.75 \mathrm{D}$ & 33.6 \\
\hline He et al.(2005) [18] & $\mathrm{SE} \leq-0.5 \mathrm{D}$ & 42.4 & NA & NA & $\mathrm{SE} \geq 2.0 \mathrm{D}$ & 1.2 & $\mathrm{SE} \geq 0.75 \mathrm{D}$ & 25.3 \\
\hline He et al.(2002) [19] & $\mathrm{SE} \leq-0.5 \mathrm{D}$ & 35.1 & NA & NA & SE $>2.0 \mathrm{D}$ & 5.8 & $\mathrm{SE} \geq 0.75 \mathrm{D}$ & 33.6 \\
\hline Lan et al. [46] & $\mathrm{SE} \leq-0.5 \mathrm{D}$ & 1 & $\mathrm{SE} \leq-6.0 \mathrm{D}$ & 0.1 & $\mathrm{SE} \geq 2.0 \mathrm{D}$ & 25.2 & $\mathrm{SE} \geq 1.5 \mathrm{D}$ & 8.2 \\
\hline Zhao et al. [49] & $\mathrm{SE} \leq-0.5 \mathrm{D}$ & 14.9 & NA & NA & $\mathrm{SE} \geq 2.0 \mathrm{D}$ & 2.6 & $\mathrm{SE} \geq 0.75 \mathrm{D}$ & 15 \\
\hline \multirow[t]{2}{*}{ Shih et al. [50] } & NA & $\mathrm{NA}$ & NA & NA & NA & NA & $\mathrm{SE} \geq 0.5 \mathrm{D}$ & 42.5 \\
\hline & NA & NA & NA & NA & NA & NA & $\mathrm{SE} \geq 0.5 \mathrm{D}$ & 51 \\
\hline \multirow{11}{*}{$\begin{array}{l}\text { Li et al. }(2006,2007,2008, \\
\text { 2009, 2010, 2011, 2012, } \\
\text { 2013, 2014, 2015) [13] }\end{array}$} & $S E<-0.5 \mathrm{D}$ & 52.02 & $\mathrm{SE} \leq-6.0 \mathrm{D}$ & 5.7 & NA & NA & NA & NA \\
\hline & $\mathrm{SE}<-0.5 \mathrm{D}$ & 55.95 & $\mathrm{SE}<-6.0 \mathrm{D}$ & 3.96 & NA & NA & NA & NA \\
\hline & $\mathrm{SE}<-0.5 \mathrm{D}$ & 56.49 & $\mathrm{SE}<-6.0 \mathrm{D}$ & 4.18 & NA & NA & NA & NA \\
\hline & $S E<-0.5 \mathrm{D}$ & 58.47 & $\mathrm{SE}<-6.0 \mathrm{D}$ & 4.75 & NA & NA & NA & NA \\
\hline & $\mathrm{SE}<-0.5 \mathrm{D}$ & 60.54 & $\mathrm{SE}<-6.0 \mathrm{D}$ & 4.98 & NA & NA & NA & NA \\
\hline & $\mathrm{SE}<-0.5 \mathrm{D}$ & 60.79 & $\mathrm{SE}<-6.0 \mathrm{D}$ & 5.52 & NA & NA & NA & NA \\
\hline & $\mathrm{SE}<-0.5 \mathrm{D}$ & 61.13 & $\mathrm{SE}<-6.0 \mathrm{D}$ & 5.89 & NA & NA & NA & NA \\
\hline & $S E<-0.5 D$ & 61.84 & $\mathrm{SE}<-6.0 \mathrm{D}$ & 5.92 & NA & NA & NA & NA \\
\hline & $\mathrm{SE}<-0.5 \mathrm{D}$ & 62.77 & $\mathrm{SE}<-6.0 \mathrm{D}$ & 6.02 & NA & NA & NA & NA \\
\hline & $S E<-0.5 \mathrm{D}$ & 63.84 & $\mathrm{SE}<-6.0 \mathrm{D}$ & 6.42 & NA & NA & NA & NA \\
\hline & $S E<-0.5 \mathrm{D}$ & 65.48 & $\mathrm{SE}<-6.0 \mathrm{D}$ & 6.69 & NA & NA & NA & NA \\
\hline
\end{tabular}

SE - spherical equivalent, D - diopters, NA - not available

*Study year are shown in the brackets for authors with the same name. 
Table 3. Subgroup analysis of refractive errors in Chinese children

\begin{tabular}{|c|c|c|c|c|c|c|c|c|c|c|c|c|c|c|c|c|}
\hline & \multicolumn{4}{|c|}{ MYOPIA } & \multicolumn{4}{|c|}{ HIGH MYOPIA } & \multicolumn{4}{|c|}{ HYPEROPIA } & \multicolumn{4}{|c|}{ ASTIGMATISM } \\
\hline & \multirow{2}{*}{$\mathrm{N}$} & \multirow{2}{*}{$\begin{array}{c}\% \\
(95 \% \text { CI })\end{array}$} & \multicolumn{2}{|c|}{ Heterogeneity } & \multirow{2}{*}{$\mathrm{N}$} & \multirow{2}{*}{$\begin{array}{c}\% \\
(95 \% \text { CI) }\end{array}$} & \multicolumn{2}{|c|}{ Heterogeneity } & \multirow{2}{*}{$\mathrm{N}$} & \multirow{2}{*}{$\begin{array}{c}\% \\
(95 \% \mathrm{CI})\end{array}$} & \multicolumn{2}{|c|}{ Heterogeneity } & \multirow{2}{*}{$\mathrm{N}$} & \multirow{2}{*}{$\begin{array}{c}\% \\
(95 \% \mathrm{CI})\end{array}$} & \multicolumn{2}{|c|}{ Heterogeneity } \\
\hline & & & $\mathrm{I}^{2}(\%)$ & $P$ & & & $I^{2}(\%)$ & $P$ & & & $\mathrm{I}^{2}(\%)$ & $\mathrm{P}$ & & & $\mathrm{I}^{2}(\%)$ & $\mathrm{P}$ \\
\hline \multicolumn{17}{|l|}{ Region: } \\
\hline Rural & 13 & $\begin{array}{c}27.1 \\
(26.7-27.5)\end{array}$ & 99.8 & $<0.001$ & 6 & $\begin{array}{c}0.9 \\
(0.3-2.9)\end{array}$ & 99.2 & $<0.001$ & 6 & $\begin{array}{c}4.0 \\
(1.3-11.4)\end{array}$ & 99.8 & $<0.001$ & 8 & $\begin{array}{c}9.9 \\
(5.9-16.0)\end{array}$ & 99.6 & $<0.001$ \\
\hline Urban & 15 & $\begin{array}{c}51.2 \\
(50.8-51.5) \\
\end{array}$ & 99.8 & $<0.001$ & 10 & $\begin{array}{c}3.0 \\
(2.3-3.8) \\
\end{array}$ & 97.7 & $<0.001$ & 11 & $\begin{array}{c}5.4 \\
(3.4-8.4) \\
\end{array}$ & 99.4 & $<0.001$ & 8 & $\begin{array}{c}20.6 \\
(15.0-27.5) \\
\end{array}$ & 99.6 & $<0.001$ \\
\hline \multicolumn{17}{|l|}{ District: } \\
\hline SC & 19 & $\begin{array}{c}31.4 \\
(31.0-31.7)\end{array}$ & 99.9 & $<0.001$ & 9 & $\begin{array}{c}0.5 \\
(0.3-0.8)\end{array}$ & 94.6 & $<0.001$ & 11 & $\begin{array}{c}10.8 \\
(10.5-11.1)\end{array}$ & 99.4 & $<0.001$ & 11 & $\begin{array}{c}12.2 \\
(8.2-17.8)\end{array}$ & 99.6 & $<0.001$ \\
\hline $\mathrm{NC}$ & 15 & $\begin{array}{c}55.1 \\
(54.9-55.3)\end{array}$ & 99.8 & $<0.001$ & 11 & $\begin{array}{c}4.6 \\
(3.6-5.7)\end{array}$ & 99.0 & $<0.001$ & 7 & $\begin{array}{c}27.8 \\
(27.1-28.6)\end{array}$ & 99.8 & $<0.001$ & 5 & $\begin{array}{c}18.5 \\
(12.7-26.1)\end{array}$ & 99.5 & $<0.001$ \\
\hline HMT & 5 & $\begin{array}{c}53.0 \\
(52.6-53.4) \\
\end{array}$ & 99.8 & $<0.001$ & 4 & $\begin{array}{c}4.9 \\
(3.5-6.9) \\
\end{array}$ & 99.1 & $<0.001$ & 2 & $\begin{array}{c}5.3 \\
(4.9-5.8) \\
\end{array}$ & 98.4 & $<0.001$ & 2 & $\begin{array}{c}35.7 \\
(20.4-54.6) \\
\end{array}$ & 99.9 & $<0.001$ \\
\hline \multicolumn{17}{|l|}{ Study year: } \\
\hline-2000 & 4 & $\begin{array}{c}49.3 \\
(37.9-60.7) \\
\end{array}$ & 99.9 & $<0.001$ & 3 & $\begin{array}{c}5.7 \\
(4.0-8.1) \\
\end{array}$ & 99.1 & $<0.001$ & 2 & $\begin{array}{c}3.2 \\
(2.1-4.9) \\
\end{array}$ & 94.9 & $<0.001$ & 3 & $\begin{array}{c}29.4 \\
(16.0-47.6) \\
\end{array}$ & 99.9 & $<0.001$ \\
\hline $2000-2010$ & 13 & $\begin{array}{c}36.5 \\
(32.6-40.5)\end{array}$ & 99.8 & $<0.001$ & 4 & $\begin{array}{c}3.2 \\
(2.4-4.2)\end{array}$ & 94.1 & $<0.001$ & 9 & $\begin{array}{c}4.1 \\
(2.2-7.4)\end{array}$ & 99.3 & $<0.001$ & 8 & $\begin{array}{c}9.5 \\
(5.2-16.7)\end{array}$ & 99.6 & $<0.001$ \\
\hline 2010- & 23 & $\begin{array}{c}36.6 \\
(31.3-42.4)\end{array}$ & 99.9 & $<0.001$ & 15 & $\begin{array}{c}2.1 \\
(1.5-2.9)\end{array}$ & 99.4 & $<0.001$ & 7 & $\begin{array}{c}5.9 \\
(3.4-9.9)\end{array}$ & 99.5 & $<0.001$ & 6 & $\begin{array}{c}18.3 \\
(13.0-25.1)\end{array}$ & 99.5 & $<0.001$ \\
\hline
\end{tabular}

$\mathrm{N}$ - number of studies, \% - pooled prevalence, SC -Southern China, NC - Northern China, HMT - Hong Kong, Macau and Taiwan

A

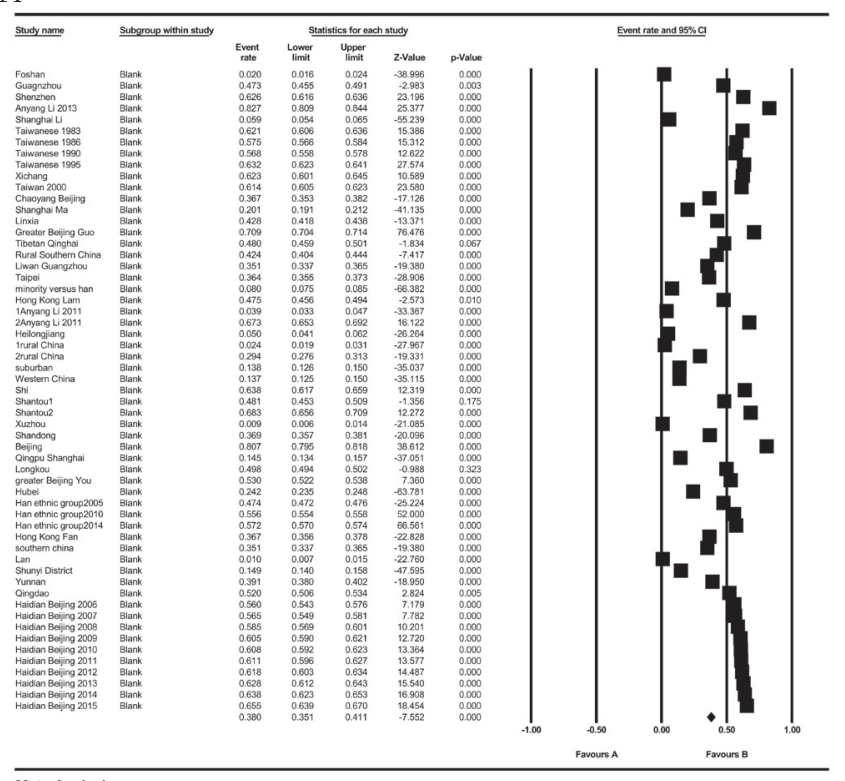

B

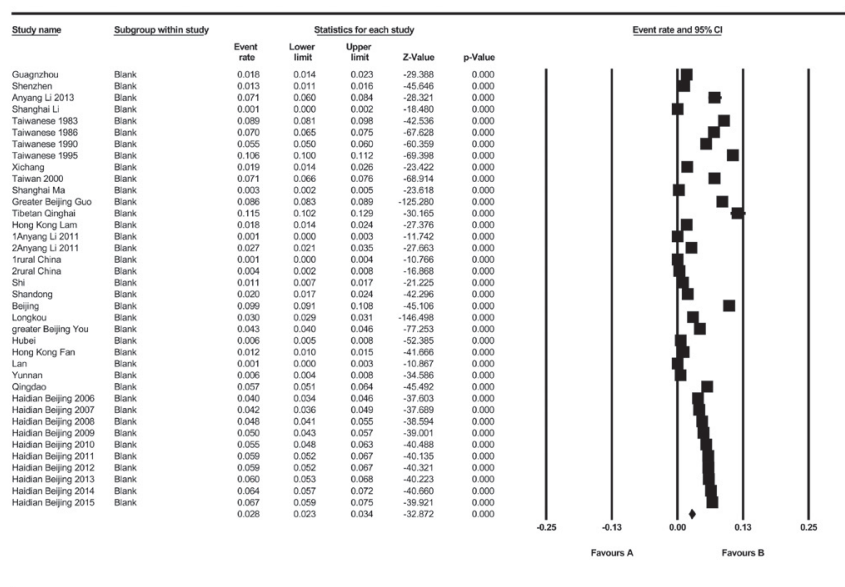

C

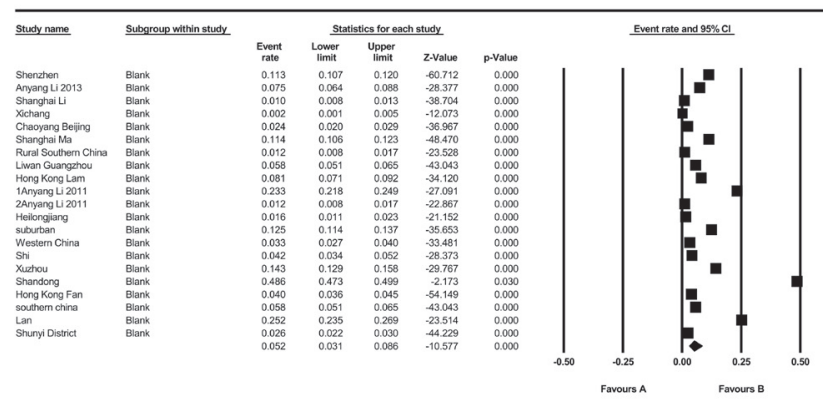

$\mathrm{D}$

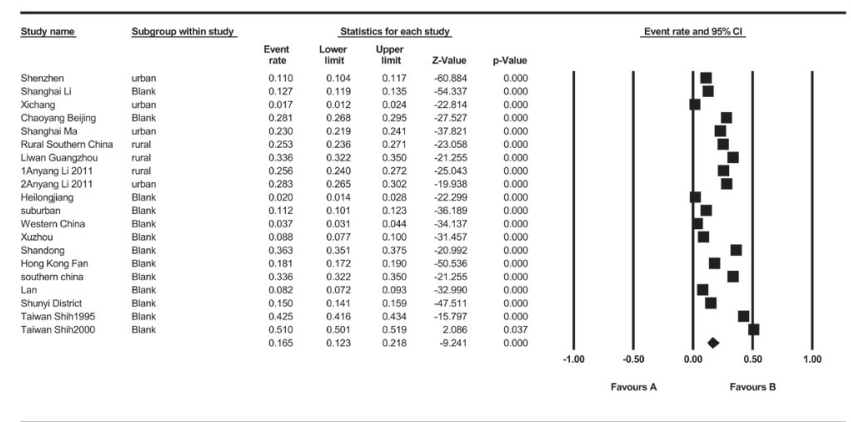

Figure 2. Meta-analysis showing the pooled prevalence of myopia (Panel A), high myopia (Panel B), hyperopia (Panel C) and astigmatism (Panel D) in Chinese children. 
Result of the meta-analysis of high myopia is presented in Figure 2, Panel B. The pooled prevalence of high myopia was $2.8 \%$ (95\% $\mathrm{CI}=2.3 \%-3.4 \%)$. There was a higher prevalence of high myopia in urban areas vs rural areas: $3.0 \%(95 \% \mathrm{CI}=2.3 \%-3.8 \%)$ vs $0.9 \%$ (95\% CI=0.3\%-2.9\%); $\mathrm{P}<0.001$. For populations in different districts, HMT and Northern China had similar pooled prevalence: $4.9 \%$ (95\% CI=3.5\%-6.9\%) vs 4.6\% (95\% CI=3.6\%-5.7\%), and Southern China still had the highest pooled prevalence: $0.5 \%$ (95\% CI=0.3\%$0.8 \%), P<0.001$. Meta regression (Figure S2 in the Online Supplementary Document) shows that the high myopia prevalence has a weak decreasing trend (equation of the regression line: high myopia prevalence $[\%]=-0.01469 \times$ midpoint of the study year group $+26.70921 ; P<0.01)$.

\section{Prevalence of hyperopia}

As shown in and Figure 2, Panel C, pooled prevalence of hyperopia was 5.2\% (95\% CI=3.1\%-8.6\%). Prevalence of hyperopia was higher in urban children than in rural children: $4.0 \%$ (95\% CI=1.3\%-11.4\%) vs 5.4\% (95\% CI $=3.4 \%-8.4 \%) ; P<0.001$. However, prevalence of hyperopia in HMT was lowest, while Northern China had the highest prevalence: $5.3 \%$ (95\% CI=4.9\%-5.8\%) vs $27.8 \%$ (95\% CI=27.1\%-28.6\%); $P<0.001$. As for the subgroup analysis of study year, prevalence of hyperopia after the year 2010 was highest while prior to the year 2000 was lowest: $5.9 \%$ (95\% CI=3.4\%-9.9\%) vs 3.2\% (95\% CI =2.1\%-4.9\%); $P<0.001$. Result of the meta regression (Figure S3 in the Online Supplementary Document) shows an increasing trend of hyperopia prevalence (hyperopia prevalence $(\%)=0.06933 \times$ midpoint of the study year group $-141.49412 ; P<0.01$ ).

\section{Prevalence of astigmatism}

Figure 2, Panel D, shows the meta-analysis results of astigmatism. The pooled prevalence of astigmatism was $16.5 \%$ ( $95 \% \mathrm{CI}=12.3 \%-21.8 \%$ ). According to the subgroup analysis by region type, prevalence of astigmatism in urban areas was dramatically higher than in rural areas: $20.6 \%(95 \% \mathrm{CI}=15 \%-27.5 \%)$ vs $9.9 \%$ (95\% $\mathrm{CI}=5.9 \% 16.0 \%) ; P<0.001$. Prevalence of astigmatism in HMT (35.7\%, 95\% CI=20.4\%-54.6\%) was highest, while prevalence in Southern China was lowest $(12.2 \%, 95 \% \mathrm{CI}=8.2 \%-17.8 \%) ; P<0.001$. Before the year 2000 the prevalence of astigmatism was highest and prevalence was lowest in the years $2000-2010(29.4 \%$, $95 \% \mathrm{CI}=16.0 \%-47.6 \%$ ) vs $47.6 \%$ (95\% CI $=5.2 \%-16.7 \%) ; P<0.001$. A decreasing trend was detected in the meta regression analysis, which is shown in Figure $S 4$ in the Online Supplementary Document (astigmatism prevalence $(\%)=-0.06604 \times$ midpoint of the study year group $+131.37988 ; P<0.01)$.

\section{Publication bias and sensitivity analysis}

According to the result of the Begg's and Egger's test, there was no publication bias detected for the prevalence of myopia, hyperopia and astigmatism $(P>0.05)$. We have also conducted the sensitivity analysis and the pooled prevalence RE did not change significantly compared with the initial results after removing each study sequentially, suggesting good homogeneity of the included studies.

\section{DISCUSSION}

In this study, 41 studies conducted in China were included for this meta-analysis and the pooled prevalence of myopia, high myopia, hyperopia, astigmatism in Chinese children were 38.0\%, 2.8\%, 5.2\%, 16.5\%, respectively. The prevalence of RE varied across different districts, region-type and period.

Compare with previous meta-analysis that reported the overall prevalence of myopia and high myopia in Chinese children from 1998 to 2016 [10], the prevalence of myopia and high myopia remains in a high level (for myopia: $37.7 \%$ vs $38 \%$, for high myopia: $3.1 \%$ vs $2.8 \%$ ), which suggest that much more efforts should be made in future to prevent and control myopia in China. Subgroup analysis in this study showed that the prevalence of myopia in urban areas is dramatically higher than that of rural areas in China. Reasons that lead to the higher prevalence of myopia in urban areas are varied, such as less outdoor activities and high academic stress $[7,8]$. Population in HMT have higher myopia prevalence in this study, which is comprehensible since these districts are highly-urbanized. According to the regression analysis, there is an increasing trend of prevalence of myopia. However, as shown in the subgroup analysis by study year, the prevalence of myopia and high myopia before the year 2000 are higher than the prevalence after the year 2010, which may be because there are fewer studies reporting prevalence before 2000 and many of them were conducted in Taiwan, a city with high prevalence of refractive errors [10,26,27,50].

It is worth noting that the increasing trend of myopia has a slowdown trend compared with the previous meta-analysis which showed a higher slope in regression analysis ( 1.086 vs 0.008$)$ and predicted that the estimat- 
ed prevalence of myopia in 2050 was 84\% [10]. This might reflected that the myopia prevention and control strategies in China [52-54], including lightening student's schoolwork burden, encouraging children to spend more time outdoors and wider use of Orthokeratology, has made some achievements in the past years. [55] Especially, the Sports for All National Strategy carried out by the government in the thirteenth Five-Year Plan of China (2016-2020), may have played an important role in myopia prevention and control in China $[56,57]$.

Comparing the prevalence of myopia in different countries, we found that prevalence of myopia and high myopia in Chinese children is significantly higher than in many other countries. For example, the prevalence of myopia ranges from 6.1\% in Morocco [58], 4.0\% in Middle East [59], to 0.8\% in Laos [60]. As for high myopia, the prevalence was $1.0 \%$ in Korea [61], and 1.4\% in India [62]. Possible explanations for this difference may lie in several aspects. First, Pan et al. revealed that factors such as higher educational level and exposure to an intensive schooling system at an early age, especially in countries such as China, are positively associated with myopia [8]; second, ethnic difference that leads to a higher prevalence in China are already discussed in many articles $[7,10,63]$. However, whether the difference between different ethnicities is caused by inter-ethnic differences in the genetic predisposition to myopia or culture-specific environmental factors still remains unclear [64].

In terms of hyperopia, as the first meta-analysis reporting prevalence of hyperopia in Chinese Children, our results show a higher prevalence was detected in urban compared to rural areas which is inconsistent with the study performed in India which showed children in rural areas were more likely to develop hyperopia than those in urban areas [65]. In our study, the higher prevalence of hyperopia in urban areas may lie in that the mean age of participants in urban areas are younger than participants in rural areas and a previous study has revealed that there is an inverse association between prevalence of hyperopia and age [66]. As for regression analysis of hyperopia, we found an increasing trend, which could be explained by the physical education reform of China (Sports for All National Strategy) which encourage children to spend more time on outdoor sports, and the policies for myopia prevention and control mentioned above $[53,54,56,57]$.

When comparing the prevalence of hyperopia with other countries and regions, prevalence of hyperopia is relatively low in China which is similar to other east Asian countries. For example, the prevalence of hyperopia is $1.5 \%$ in Singapore and $6.2 \%$ in Korea $[61,67]$. In contrast, prevalence is higher in western countries, from $13.1 \%$ in Poland to $14.7 \%$ in Northern Ireland $[68,69]$. Both environmental factors and ethnic and genetic factors may contribute to the low prevalence of hyperopia in China. For the environmental factors, as is mentioned above, spending more time outdoors and living in rural areas often leads to hyperopia while children in China tend to spend less time outdoors and more time in near work $[65,70]$. As for the ethnic factor of hyperopia, the CLEERE study reported that Caucasians had the highest prevalence of hyperopia while Asians have the lowest prevalence [63,66]. According to the meta-analysis by Hashemi et al [6], genetic and ethnic factors could play a more prominent role in hyperopia.

The prevalence of astigmatism in Chinese children is $16.5 \%$ and highest prevalence was seen in HMT (35.7\%), which is higher than many countries. For example, prevalence was only $5.4 \%$ in India, $6.7 \%$ in Australia and $9 \%$ in Laos $[60,62,71]$. As is mentioned earlier, near work is one of the major reason that leads to astigmatism, and the high stress on academic performance may contribute to the high prevalence of astigmatism in China $[72,73]$. Ethnicity also plays an important part in the prevalence of astigmatism. As is reported in the CLEERE study, Asians and Hispanics had the highest prevalence of astigmatism [63], which may be explained by the anatomy of Asian eyes (tight eyelids and narrow palpebral apertures) [74]. When compared our results with the study conducted in Taiwan, which reported a prevalence of astigmatism was 42.5\% in 1995 and $51 \%$ in 2000 , an obvious decreasing trend was suggested. A reasonable explanation might be the perform of series policies, including the myopia prevent strategies mentioned above [72]. In the subgroup analysis by region, a higher astigmatism prevalence was detected in urban regions vs rural regions. One explanation could be urban children are engaging in more near work, and as a former study reported not only can near work cause myopia but it is also likely to increase the risk of astigmatism [72]. In the subgroup analysis by district, the highest astigmatism prevalence was found in HMT, which are highly urbanization areas, which is consistent with subgroup analysis of region-type.

This study has several limitations. First, few studies were prior to the 2000 or in remote provinces which will affect the precision of the results to a certain extent. Second, not all the studies used the same definition of RE which might influence the result in some extent. Third, out of 40 studies, 9 of them did not perform cycloplegia for refractometry which might also influence the result. Nevertheless, to the best of our knowledge, this is the first meta-analysis to report the overall prevalence and time trend analysis of refractive errors and its sub-classifications in Chinese children. Additionally, this article includes numerous studies throughout China, covering a large population with a wide geographical distribution. 


\section{CONCLUSION}

The pooled prevalence of myopia, high myopia, hyperopia, astigmatism in Chinese children are $38.0 \%, 2.8 \%$, $5.2 \%, 16.5 \%$, respectively. Urban children are more vulnerable to RE (especially myopia) than rural children. Children living in HMT have a higher prevalence of myopia, high myopia and astigmatism than children in mainland China. There is an increasing trend for prevalence of myopia and hyperopia while there is a decreasing trend for prevalence of high myopia and astigmatism in Chinese children. Considering the large magnitude of refractive errors, more attention should be paid to RE prevention and treatment strategy development in China.

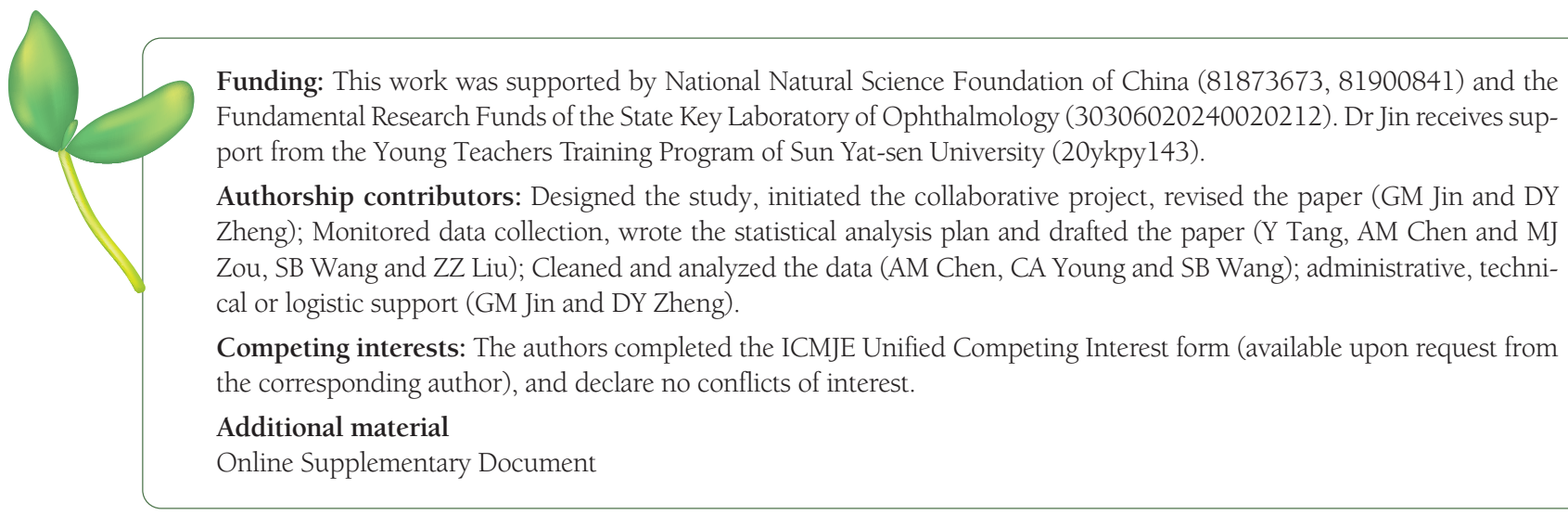

1 Pascolini D, Mariotti SP. Global estimates of visual impairment: 2010. Br J Ophthalmol. 2012;96:614-8. Medline:22133988 doi:10.1136/bjophthalmol-2011-300539

2 Ohno-Matsui K, Kawasaki R, Jonas JB, Cheung CM, Saw SM, Verhoeven VJ, et al. International photographic classification and grading system for myopic maculopathy. Am J Ophthalmol. 2015;159:877-83 e7. Medline:25634530 doi:10.1016/j. ajo.2015.01.022

3 Packwood EA. The psychosocial effects of amblyopia study. J AAPOS. 1999;3(1):15-7. Medline:10071896 doi:10.1016/S10918531(99)70089-3

4 Naidoo KS, Jaggernath J. Uncorrected refractive errors. Indian J Ophthalmol. 2012;60:432-7. Medline:22944755 doi:10.4103/0301-4738.100543

5 Morgan IG, Ohno-Matsui K, Saw S-M. Myopia. Lancet. 2012;379:1739-48. Medline:22559900 doi:10.1016/S01406736(12)60272-4

6 Hashemi H, Fotouhi A, Yekta A, Pakzad R, Ostadimoghaddam H, Khabazkhoob M. Global and regional estimates of prevalence of refractive errors: Systematic review and meta-analysis. J Curr Ophthalmol. 2017;30:3-22. Medline:29564404 doi:10.1016/j. joco.2017.08.009

7 Pan CW, Cheng C-Y. The Age-Specific Prevalence of Myopia in Asia: A Meta-analysis. Optom Vis Sci. 2015;92(3):258-66. Medline:25611765

8 Pan CW, Ramamurthy D, Saw SM. Worldwide prevalence and risk factors for myopia. Ophthalmic Physiol Opt. 2012;32:316. Medline:22150586 doi:10.1111/j.1475-1313.2011.00884.x

9 Holden BA, Fricke TR, Wilson DA, Jong M, Naidoo KS, Sankaridurg P, et al. Global Prevalence of Myopia and High Myopia and Temporal Trends from 2000 through 2050. Ophthalmology. 2016;123:1036-42. Medline:26875007 doi:10.1016/j.ophtha.2016.01.006 Medline:29121045 doi:10.1371/journal.pone.0187396

10 Dong L, Kang YK, Li Y, Wei WB, Jonas JB. Prevalence and time trends of myopia in children and adolescents in China: a systemic review and meta-analysis. Retina. 2019;40:399-441.

11 Guo Y, Duan JL, Liu LJ, Sun Y, Tang P, Lv YY, et al. High myopia in Greater Beijing School Children in 2016. PLoS One. 2017;12:e0187396. Medline:29121045 doi:10.1371/journal.pone.0187396

12 Lam CS, Lam CH, Cheng SC, Chan LY. Prevalence of myopia among Hong Kong Chinese schoolchildren: changes over two decades. Ophthalmic Physiol Opt. 2012;32:17-24. Medline:22150587 doi:10.1111/j.1475-1313.2011.00886.x

13 Li Y, Liu J, Qi P. The increasing prevalence of myopia in junior high school students in the Haidian District of Beijing, China: a 10-year population-based survey. BMC Ophthalmol. 2017;17:88. Medline:28606071 doi:10.1186/s12886-017-0483-6

14 Guo JL, Huang XL, Yu FC, Liang SZ, Lai TT. Epidemiological survey of preschool children with myopia in foshan and the analysis of related factors. Int J Ophthalmol Clin Res. 2010;10:1191-2.

15 Guo L, Yang J, Mai J, Du X, Guo Y, Li P, et al. Prevalence and associated factors of myopia among primary and middle school-aged students: A school-based study in Guangzhou. Eye (Lond). 2016;30:796-804. Medline:26965016 doi:10.1038/ eye.2016.39

16 Han B, Zhou WW, Liu CM, Yang Y, Cheng HB, Xu XJ. Epidemiological study on visual acuity and refractive status of primary students and junior high school students in Shenzhen. Int J Ophthalmol Clin Res. 2016;16:2103-6.

17 Han X, Miao HL, Huang D. Investigation of junior school student myopia in high-altitude Tibetan areas in Qinghai Province. Int J Ophthalmol Clin Res. 2014;14:913-5. 
18 He M, Huang W, Zheng Y, Huang L, Ellwein LB. Refractive Error and Visual Impairment in School Children in Rural Southern China. Ophthalmology. 2007;114:374-82. Medline:17123622 doi:10.1016/j.ophtha.2006.08.020

19 He MG, Lin Z, Huang J, Lu Y, Wu CF, Xu JJ. Population-based survey of refractive error in school-aged children in Liwan District, Guangzhou. Zhonghua Yan Ke Za Zhi. 2008;44:491-6. Medline:19035237

20 Hsu CC, Huang N, Lin PY, Tsai DC, Tsai CY, Woung LC, et al. Prevalence and risk factors for myopia in second-grade primary school children in Taipei: A population-based study. J Chin Med Assoc. 2016;79:625-32. Medline:27349942 doi:10.1016/j. jcma.2016.02.011

21 Hu M, Zhou Y, Huang S, Congdon N, Jin L, Wang X, et al. Population prevalence of myopia, glasses wear and free glasses acceptance among minority versus han schoolchildren in China. PLoS One. 2019;14:e0215660. Medline:30998750 doi:10.1371/ journal.pone.0215660

22 Li H, Li S, Liu L, Li S, Kang M, Ji Y, et al. Distribution of refraction and ocular biometric parameters in a population of junior middle school children in Anyang of Henan province. Zhonghua Yi Xue Za Zhi. 2014;94:1284-8. Medline:25142845

23 Li SM, Liu LR, Li SY, Ji YZ, Fu J, Wang Y, et al. Design, methodology and baseline data of a school-based cohort study in central china: The anyang childhood eye study. Ophthalmic Epidemiol. 2013;20:348-59. Medline:24160405 doi:10.3109/092 86586.2013 .842596

24 Li T, Zhou X, Chen X, Qi H, Gao Q. Refractive Error in Chinese Preschool Children: The Shanghai Study. Eye Contact Lens. 2019;45:182-7. Medline:30260815 doi:10.1097/ICL.0000000000000555

$25 \mathrm{Li}$ Z, Xu K, Wu S, Lv J, Jin D, Song Z, et al. Population-based survey of refractive error among school-aged children in rural northern China: The Heilongjiang eye study. Clin Exp Ophthalmol. 2014:42:379-84. Medline:23952961 doi:10.1111/ceo.12198

26 Lin LLK, Shih YF, Hsiao CK, Chen CJ. Prevalence of Myopia in Taiwanese Schoolchildren: 1983 to 2000. Ann Acad Med Singap. 2004;33:27-33. Medline:15008558

27 Lin LLK, Shih YF, Hsiao CK, Chen CJ, Lee LA, Hung PT. Epidemiologic study of the prevalence and severity of myopia among schoolchildren in Taiwan in 2000. J Formos Med Assoc. 2001;100:684-91. Medline:11760374

28 Lyu Y, Zhang H, Gong Y, Wang D, Chen T, Guo X, et al. Prevalence of and factors associated with myopia in primary school students in the Chaoyang District of Beijing, China. Jpn J Ophthalmol. 2015;59:421-9. Medline:26329826 doi:10.1007/ s10384-015-0409-X

29 Ma Y, Qu X, Zhu X, Xu X, Zhu J, Sankaridurg P, et al. Age-specific prevalence of visual impairment and refractive error in children aged 3-10 years in Shanghai, China. Invest Ophthalmol Vis Sci. 2016;57:6188-96. Medline:27842160 doi:10.1167/ iovs. 16-20243

30 Pan CW, Wu RK, Li J, Zhong H. Low prevalence of myopia among school children in rural China. BMC Ophthalmol. 2018;18:140. Medline:29890943 doi:10.1186/s12886-018-0808-0

31 Pi LH, Chen L, Liu Q, Ke N, Fang J, Zhang S, et al. Refractive status and prevalence of refractive errors in suburban school-age children. Int J Med Sci. 2010;7:342-53. Medline:20975844 doi:10.7150/ijms.7.342

32 Pi LH, Chen L, Liu Q, Ke N, Fang J, Zhang S, et al. Prevalence of eye diseases and causes of visual impairment in school-aged children in Western China. J Epidemiol. 2012;22:37-44. Medline:22123227 doi:10.2188/jea.JE20110063

33 Qian ML, Li ZH, Bai HL, Kong BH, Xie L, Liu Q. Prevalence and related factors investigations of myopia among multi-ethnic adolescents in Linxia Prefecture. Int J Ophthalmol Clin Res. 2018;18:1105-8.

34 Shi Y, Hu J, Zhang C, Sun Y, Shi R, Li J, et al. Analysis of refractive status without cycloplegia in 2046 Pupils. Yan ke xue bao. 2010;25:16-9. Medline:21166033

35 Wang G, Tan L, Huang YQ, Lin SB, Chen B, Zhang MZ. A cross-sectional study of myopia among students from middle and high schools in Shantou, China. Int J Ophthalmol Clin Res. 2014;14:1491-3.

36 Wang X, Liu D, Feng R, Zhao H, Wang Q. Refractive error among urban preschool children in Xuzhou, China. Int J Clin Exp Pathol. 2014;7:8922-8. Medline:25674266

$37 \mathrm{Wu}$ JF, Bi HS, Wang SM, Hu YY, Wu H, Sun W, et al. Refractive error, visual acuity and causes of vision loss in children in Shandong, China. The Shandong children eye study. PLoS One. 2013;8:e82763. Medline:24376575 doi:10.1371/journal. pone.0082763

38 Wu LJ, You QS, Duan JL, Luo YX, Liu LJ, Li X, et al. Prevalence and associated factors of myopia in high-school students in Beijing. PLoS One. 2015;10:e0120764. Medline:25803875 doi:10.1371/journal.pone.0120764

39 Xia W, Gong LH, Zhang XY, Zhang AH, Miao L. Investigation analysis of children myopia in Qingpu district of Shanghai. Int J Ophthalmol Clin Res. 2010;10:2157-8.

40 Yang XW, Qu GZ, Liu LM, Wang N, Li H, Xing L, et al. Prevalence of myopia among primary and middle school students in Longkou City, Shandong Province. Int J Ophthalmol Clin Res. 2018;18:1287-9.

41 You QS, Wu LJ, Duan JL, Luo YX, Liu LJ, Li X, et al. Prevalence of myopia in school children in greater Beijing: The Beijing Childhood Eye Study. Acta Ophthalmol. 2014;92:e398-406. Medline:25165786 doi:10.1111/aos.12299

42 Zeng CQ, Zhou LH, Zhang P, Wang J, Ye MH, Yi BX, et al. The epidemiology of myopia in primary school students of grade 1 to 3 in Hubei province. Zhonghua Yan Ke Za Zhi. 2018;54:756-61. Medline:30347563

43 Congdon N, Wang Y, Song Y, Choi K, Zhang M, Zhou Z, et al. Visual disability, visual function, and myopia among rural chinese secondary school children: the Xichang Pediatric Refractive Error Study (X-PRES)-report 1. Invest Ophthalmol Vis Sci. 2008;49:2888-94. Medline:18378579 doi:10.1167/iovs.07-1160

44 Fan DS, Lam DS, Lam RF, Lau JT, Chong KS, Cheung EY, et al. Prevalence, incidence, and progression of myopia of school children in Hong Kong. Invest Ophthalmol Vis Sci. 2004:45:1071-5. Medline:15037570 doi:10.1167/iovs.03-1151

45 He M, Zeng J, Liu Y, Xu J, Pokharel GP, Ellwein LB. Refractive error and visual impairment in urban children in southern china. Invest Ophthalmol Vis Sci. 2004;45:793-9. Medline:14985292 doi:10.1167/iovs.03-1051 
46 Lan W, Zhao F, Lin L, Li Z, Zeng J, Yang Z, et al. Refractive errors in 3-6 year-old Chinese children: a very low prevalence of myopia? PLoS One. 2013;8:e78003. Medline:24205064 doi:10.1371/journal.pone.0078003

47 Qian DJ, Zhong H, Li J, Niu Z, Yuan Y, Pan CW. Myopia among school students in rural China (Yunnan). Ophthalmic Physiol Opt. 2016;36:381-7. Medline:26896871 doi:10.1111/opo.12287

48 Sun JT, An M, Yan XB, Li GH, Wang DB. Prevalence and Related Factors for Myopia in School-Aged Children in Qingdao. J Ophthalmol. 2018;2018:9781987. Medline:29507811 doi:10.1155/2018/9781987

49 Zhao J, Pan X, Sui R, Muoz SR, Sperduto RD, Ellwein LB. Refractive Error Study in Children: Results From Shunyi District, China. Am J Ophthalmol. 2000;129:427. Medline:10764849 doi:10.1097/00006324-200402000-00007

50 Shih YF, Hsiao CK, Tung YL, Lin LLK, Chen CJ, Hung PT. The prevalence of astifmatism in Taiwan schoolchildren. Optom Vis Sci. 2004;81:94-8. Medline:15127928 doi:10.1016/s0002-9394(99)00452-3

51 Dong YH, Liu HB, Wang ZH, Xu RB, Yang ZP, Ma J. The epidemic status and secular trends of myopia prevalence for Chinese children and adolescents aged 7-18 years from 2005 to 2014. Zhonghua Yu Fang Yi Xue Za Zhi. 2017;51:285-9. Medline:28395459

52 Yu Juan YZ, Song B, Jiang S. Research Progress of juvenile myopia prevention and control. J Nurs Sci. 2014;29:86.

$53 \mathrm{Ma} \mathrm{J}$. Pay attention to implementing measures, promote prevention and control of myopia. Zhonghua Yu Fang Yi Xue Za Zhi. 2021;55:440-5. Medline:33858053 doi:10.3760/cma.j.cn112150-20210223-00178

54 Liao W. The basic principle of prevention and control of adolescent myopia. Zhongguo Xue Xiao Wei Sheng. 2008;29:385-7.

55 Bullimore MA, Johnson LA. Overnight orthokeratology. Cont Lens Anterior Eye. 2020;43:322-32. Medline:32331970 doi:10.1016/j.clae.2020.03.018

$56 \mathrm{Hu}$ AG, Fang XD. The development idea of sports for all national strategy. Ti Yu Ke Xue. 2016;36:3.

57 Ren H, Wang KZ, Xiao SH, Zhao SX. On format for sport resources distribution - Sport reform under the social-economic transformation in China. Journal of Tianjin Institute of Physical Education. 2001;16:2.

58 Anera RG, Soler M, de la Cruz Cardona J, Salas C, Ortiz C. Prevalence of refractive errors in school-age children in Morocco. Clin Exp Ophthalmol. 2009;37:191-6. Medline:19723127 doi:10.1111/j.1442-9071.2009.02001.x

59 Khoshhal F, Hashemi H, Hooshmand E, Saatchi M, Yekta A, Aghamirsalim M, et al. The prevalence of refractive errors in the Middle East: a systematic review and meta-analysis. Int Ophthalmol. 2020;40(6):1571-86. Medline:32107693 doi:10.1007/ s10792-020-01316-5

60 Casson RJ, Kahawita S, Kong A, Muecke J, Sisaleumsak S, Visonnavong V. Exceptionally low prevalence of refractive error and visual impairment in schoolchildren from Lao People's Democratic Republic. Ophthalmology. 2012;119:2021-7. Medline:22698982 doi:10.1016/j.ophtha.2012.03.049

61 Jang JU, Park IJ. The status of refractive errors in elementary school children in South Jeolla Province, South Korea. Clin Optom (Auckl). 2015;7:45-51. doi:10.2147/OPTO.S85992

62 Sheeladevi S, Seelam B, Nukella PB, Modi A, Ali R, Keay L. Prevalence of refractive errors in children in India: a systematic review. Clin Exp Optom. 2018;101:495-503. Medline:29682791 doi:10.1111/cxo.12689

63 Kleinstein RN, Jones LA, Hullet S, Kwon S, Lee RJ, Friedman NE, et al. Refractive Error and Ethnicity in Children. Arch Ophthalmol. 2003;121:1141-7. Medline:12912692 doi:10.1111/j.1399-0004.2010.01592.x

64 Wojciechowski R. Nature and nurture: the complex genetics of myopia and refractive error. Clin Genet. 2011;79:301-20. Medline:21155761 doi:10.1001/archopht.121.8.1141

65 Dandona R, Dandona L, Srinivas M, Giridhar P, McCarty CA, Rao GN. Population-based assessment of refractive error in India: the Andhra Pradesh eye disease study. Clin Exp Ophthalmol. 2002;30:84-93. Medline:11886410 doi:10.1046/j.14426404.2002.00492.x

66 Castano VD, Fassa AG, Carret MLV, Vilela MAP, Meucci RD. Hyperopia: a meta-analysis of prevalence and a review of associated factors among school-agedchildren. BMC Ophthalmol. 2014;14:163. Medline:25539893 doi:10.1186/1471-2415-14-163

67 Quek TPL, Chua CS, Chong JH, Hey HW, Lee J, Lim YF, et al. Prevalence of Refractive Errors in Teenage High School Students in Singapore. Ophthalmic Physiol Opt. 2004;24:47-55. Medline:14687201 doi:10.1046/j.1475-1313.2003.00166.x

68 Czepita D, Zejmo M, Mojsa A. Prevalence of myopia and hyperopia in a population of Polish schoolchildren. Ophthalmic Physiol Opt. 2007;27:60-5. Medline:17239191 doi:10.1111/j.1475-1313.2006.00419.x

69 O'Donoghue L, McClelland JF, Logan NS, Rudnicka AR, Owen CG, Saunders KJ. Refractive error and visual impairment in school children in Northern Ireland. Br J Ophthalmol. 2010;94:1155-9. Medline:20494909 doi:10.1136/bjo.2009.176040

70 Rose KA. Outdoor activity reduces the prevalence of myopia in children. Ophthalmology. 2008;8:1279. Medline:18294691 doi:10.1016/j.ophtha.2007.12.019

71 Huynh SC, Kifley A, Rose KA, Morgan IG, Mitchell P. Astigmatism in 12-year-old Australian children: comparisons with a 6-year-old population. Invest Ophthalmol Vis Sci. 2007;48:73-82. Medline:17197519 doi:10.1167/iovs.06-0263

72 Buehren T, Collins MJ, Loughridge J, Carney LG, Iskander DR. Corneal topography and accommodation. Cornea. 2003;22:3116. Medline:12792473 doi:10.1097/00003226-200305000-00007

73 Gwiazda J, Grice K, Held R, McLellan J, Thorn F. Astigmatism and the development of myopia in children. Vision Res. 2000;40:1019-26. doi:10.1016/S0042-6989(99)00237-0

74 Kame RT, Jue TS, Shigekuni DM. A longitudinal study of corneal astigmatism changes in Asian eyes. J Am Optom Assoc. 1993;64:215-9. Medline:8454841 